\title{
О РЕШЕНИИ НЕЛИНЕЙНЫХ УРАВНЕНИЙ МЕТОДАМИ ВЫСОКОГО ПОРЯДКА СХОДИМОСТИ И ИХ УСТОИЧИВОСТИ
}

При решении нелинейных уравнений на ЭВМ итерационными методами за меру эффективности последних обычно принимают индекс эффективности Островского [ $\left.{ }^{1}\right]$ или его обобщенно-уточненные варианты $\left[{ }^{2-4}\right]$, использующие порядок сходимости $p$ метода и объем вычислений на каждом итерационном шаге. Следует, однако, отметить, что при определении этого объема, как правило, не учитывается чувствительность применяемого метода к ошибкам округлений, в том числе и к ошибкам, возникающим при решении линейных задач на каждом шаге итерации. В данной работе на примере одного класса итерационных методов четвертого порядка сходимости устанавливается, в какой же мере ошибки, появляющиеся при решении линейных задач, влияют на порядок его сходимости.

1. Пусть $F(x)$ - дифференцируемый необходимое число раз нелинейный оператор из одного банахова пространства $X$ в другое $Y$.

Для решения уравнения

$$
F(x)=0
$$

рассмотрим следующий класс итерационных методов

$$
\begin{aligned}
& x_{k+1}=v_{k}-2 A_{k} F\left(v_{k}\right)+\frac{1}{\varrho} A_{k}\left[F\left(v_{k}+\varrho A_{k} F\left(v_{k}\right)\right)-F\left(v_{k}\right)\right], \\
& v_{k}=x_{k}-\tilde{A}_{k} F\left(x_{k}\right), \quad k=0,1, \ldots,
\end{aligned}
$$

где $A_{k}$ и $\bar{A}_{k}-$ некоторые аппроксимащии для $\Gamma_{k}=\left[F^{\prime}\left(x_{k}\right)\right]^{-1}$, $\left\|I-F^{\prime}\left(x_{k}\right) A_{k}\right\| \leqslant \gamma_{k}<1,\left\|I-F^{\prime}\left(x_{k}\right) \tilde{A}_{k}\right\| \leqslant \tilde{\gamma}_{k}<1$ и $е-$ произвольный параметр, не равный нулю (I - единичный оператор).

При $A_{k}=\widetilde{A}_{k}=\Gamma_{k}$ получим обобщение известного класса итерационных методов, предложенного Дж. Траубом $\left[{ }^{2}\right]$ для решения одного вещественного нелинейного уравнения с одним неизвестным $-f(x)=0$ на случай банахова пространства. Дж. Трауб выделил из этого класса наиболее простые варианты, получаемые при $\varrho=-1$ (см. (6)) и $\varrho=$ $=-1 / 2$ и, кроме того, доказал, что весь этот класс обеспечивает в общем скорость сходимости к решению $f(x)=0$ четвертого порядка и в одном случае, при $\varrho=-5$, пятого порядка. Но поскольку последний вариант нам не удалось реализовать из-за некоммутативности операторов, появляющихся в используемых нами оценках, будем считать его однопараметрическим классом методов четвертого порядка сходимости. Кроме того, при $\varrho \rightarrow 0$ придем к методу, рассмотренному в [ $\left.{ }^{5}\right]$. Итак, данную работу можно рассматривать как обобщение и продолжение ра. боты $\left[{ }^{5}\right]$. 
Предположим, что операторы $A_{k}, \widetilde{A}_{k}, F^{\prime}(x), F^{\prime \prime}(x), F^{\prime \prime \prime}(x)$ ограничены по норме, а третья производная оператора $F(x)$ удовлетворяет условию Липшица в некоторой области $S$ из $X$. Тогда нетрудно показать (см. $\left.\left[{ }^{5}\right]\right)$, что

$$
\begin{aligned}
\left\|F\left(x_{k+1}\right)\right\| \leqslant & K_{1} \hat{\gamma}_{k}^{2} \tilde{\gamma}_{k}\left\|F\left(x_{k}\right)\right\|+\left(K_{2} \hat{\gamma}_{k}^{2}+K_{3} \tilde{\gamma}_{k} \tilde{\gamma}_{k}+K_{4} \tilde{\gamma}_{k}^{2}\right)\left\|F\left(x_{k}\right)\right\|^{2}+ \\
& +\left(K_{5} \gamma_{k}+K_{6} \tilde{\gamma_{k}}\right)\left\|F\left(x_{k}\right)\right\|^{3}+K_{7}\left\|F\left(x_{k}\right)\right\|^{4}+R,
\end{aligned}
$$

где $K_{i}(i=1, \ldots, 7)$ некоторые константы и $R$ содержит члены более высокого порядка, т. е. члены вида $O\left(\gamma_{k}^{r} \tilde{\gamma}_{k}^{s}\left\|F\left(x_{k}\right)\right\|^{t}\right), r+s+t \geqslant 5$.

Решение уравнения (1) методами типа (2) равносильно решению на каждом шаге итерации возмущенных линейных уравнений

$$
\begin{gathered}
{\left[F^{\prime}\left(x_{k}\right)+\widetilde{V}_{k}\right] t_{k}=-F\left(x_{k}\right),} \\
{\left[F^{\prime}\left(x_{k}\right)+V_{k}\right] \zeta_{k}=F\left(v_{k}\right),} \\
{\left[F^{\prime}\left(x_{k}\right)+V_{k}\right] w_{k}=1 / \mathrm{Q}\left[F\left(v_{k}+\mathrm{Q} \zeta_{k}\right)-F\left(v_{k}\right)\right]-2 F\left(v_{k}\right),}
\end{gathered}
$$

где $t_{k}=v_{k}-x_{k}, \widetilde{V}_{k}=\tilde{A}_{k}^{-1}-F^{\prime}\left(x_{k}\right)$ и $V_{k}=A_{k}^{-1}-F^{\prime}\left(x_{k}\right)$, и поэтому изучение сходимости класса методов (2) позволяет судить об устойчивости основного класса методов $\left(A_{k}=\widetilde{A}_{k}=\Gamma_{k}\right)$. При $\gamma_{k}=O\left(\left\|F\left(x_{k}\right)\right\|\right)$ и $\bar{\gamma}_{k}=O\left(\left\|F\left(x_{k}\right)\right\|\right) \quad$ (или $\left\|V_{k}\right\|=O\left(\left\|F\left(x_{k}\right)\right\|\right)$ и $\left.\left\|\widetilde{V}_{k}\right\|=O\left(\left\|F\left(x_{k}\right)\right\|\right) \quad\left[{ }^{5}\right]\right)$ на основании оценки (3) заключаем, что класс методов (2) имеет такой же порядок сходимости, что и основной, то есть четвертый.

Если же за $A_{k}$ принять $F^{-1}\left(\tilde{x}_{k} ; x_{k}\right)$, а за $\widetilde{A}_{k}$ соответственно $F^{-1}\left(\hat{x}_{k} ; x_{k}\right)$, где $F(\cdot ;)$ - разделенная разность первого порядка оператора $F(x)\left[{ }^{6}\right]$, то нетрудно видеть, что сохранение четвертого порядка сходимости у методов (2) гарантируется выбором $\tilde{x}_{k}$ и $\hat{x}_{k}$ из условий

$$
\begin{aligned}
& \left\|\tilde{x}_{k}-x_{k}\right\| \leqslant C_{k}\left\|F\left(x_{k}\right)\right\|, \\
& \left\|\hat{x}_{k}-x_{k}\right\| \leqslant C_{k}\left\|F\left(x_{k}\right)\right\|
\end{aligned}
$$

или

$$
\begin{aligned}
& \tilde{x}_{k}=x_{k}-\varepsilon_{k} S_{k} F\left(x_{k}\right), \\
& \hat{x}_{k}=x_{k}-\tilde{\varepsilon}_{k} \widetilde{S}_{k} F\left(x_{k}\right),
\end{aligned}
$$

где $\varepsilon_{k}, \tilde{\varepsilon_{k}}>0, S_{k}, \tilde{S}_{k} \in[Y \rightarrow X], C_{k}, \tilde{C}_{k}-$ некоторые постоянные. Однако условия (5) практически труднореализуемы из-за отсутствия общих правил выбора $C_{k}, \tilde{C}_{k}, \varepsilon_{k}, \tilde{\varepsilon}_{k}$.

Покажем два варианта практически удобных выражений для $A_{k}$ и $\widetilde{A}_{k}$, не требующих для реализации выбора дополнительных параметров:

1) $A_{k}=\widetilde{A}_{k}=F^{-1}\left(2 x_{k}-x_{k-1} ; x_{k-1}\right)$.

Предполагая в дальнейшем, что разделенные разности оператора $F(x)$ до третьего порядка включительно ограничены в $S$, получим

$$
\text { 2) } \begin{aligned}
\gamma_{k} & =\tilde{\gamma}_{k}=O\left(\left\|F\left(x_{k-1}\right)\right\|^{2}\right) . \\
A_{k} & =F^{-1}\left(2 v_{k}-x_{k} ; x_{k}\right), \\
\tilde{A}_{k} & =F^{-1}\left(2 v_{k-1}-x_{k-1} ; x_{k-1}\right) .
\end{aligned}
$$


Тогда

$$
\begin{aligned}
& \gamma_{k}=O\left(\left\|F\left(x_{k}\right)\right\|\right), \\
& \tilde{\gamma}_{k}=O\left(\left\|F\left(x_{k-1}\right)\right\|\left\|F\left(x_{k-2}\right)\right\| \ldots\left\|F\left(x_{0}\right)\right\|\right) .
\end{aligned}
$$

Выделяя в неравенстве (3) для этих вариантов доминирующие члены $O\left(\left\|F\left(x_{k}\right)\right\|\left\|F\left(x_{k-1}\right)\right\|^{6}\right) \quad$ и $O\left[\left(\left\|F\left(x_{k}\right)\right\|\left\|F\left(x_{k-1}\right)\right\| \ldots\left\|F\left(x_{0}\right)\right\|\right)^{2}\right]$, видим, что их асимптотические порядки сходимости равны трем $\left[{ }^{7}\right]$. Следовательно, решение линейных уравнений основного класса методов с погрешностями $\gamma_{k}$ и $\tilde{\gamma}_{k}$ приведет к тому, что его порядок сходимости упадет сразу на единицу. Отметим, что в обоих вариантах на каждом итерационном шаге (за исключением первого) вычисляются, кроме линейных задач (4), три значения оператора $F(x)$ и одна первая разделенная разность. В то время как основной класс методов требует вычисления трех значений оператора и одного значения производной.

Допустим теперь, что $F\left(\widetilde{x}_{k} ; x_{k}\right)=F_{k}$ вычислена также с некоторой погрешностью $\Delta F_{k}$. Тогда, принимая $A_{k}=\tilde{A}_{k}=\left[F_{k}+\Delta F_{k}\right]^{-1}$, имеем

$$
\begin{array}{r}
\left\|I-F^{\prime}\left(x_{k}\right) A_{k}\right\|=\left\|I-F^{\prime}\left(x_{k}\right)\left[F_{k}+\Delta F_{k}\right]^{-1}\right\| \leqslant \\
\leqslant\left\|F_{k}+\Delta F_{k}-F^{\prime}\left(x_{k}\right)\right\|\left\|\left[F_{k}+\Delta F_{k}\right]^{-1}\right\| .
\end{array}
$$

Поэтому, если $\left\|F_{k}-F^{\prime}\left(x_{k}\right)\right\|=O\left(\left\|F\left(x_{k}\right)\right\|\right)$, то условие $\left\|\Delta F_{k}\right\|=$ $=O\left(\left\|F\left(x_{k}\right)\right\|\right)$ достаточно для того, чтобы $\left\|F_{k}-F^{\prime}\left(x_{k}\right)+\Delta F_{k}\right\|=$ $=O\left(\left\|F\left(x_{k}\right)\right\|\right) \quad$ и, следовательно, $\left\|I-F^{\prime}\left(x_{k}\right) A_{k}\right\|=O\left(\left\|F\left(x_{k}\right)\right\|\right)$, т. е. если неточность $\Delta F_{k}$ вычисления $F_{k}$ не превысит $O\left(\left\|F\left(x_{k}\right)\right\|\right)$ на каждом итерационном шаге, то порядок сходимости класса методов (2) будет равен четырем.

Пр и мечание. Различные варианты, которым метод, получаемый из класса (2) при $\varrho \rightarrow 0$, обеспечивает скорость сходимости третьего порядка $\left[{ }^{5}\right]$, можно дополнить модификацией

$$
\begin{aligned}
& A_{k}=B_{k}^{-1}=F^{-1}\left(2 v_{k}-x_{k} ; x_{k}\right), \\
& \widetilde{A}_{k}=F^{-1}\left(2 v_{k-1}-x_{k-1} ; x_{k-1}\right),
\end{aligned}
$$

где $B_{k}$ является аппроксимацией оператора $F^{\prime}\left(v_{k}\right)$. В таком случае метод, рассмотренный в $\left[{ }^{5}\right]$, примет более простой вид

$$
\begin{gathered}
x_{k+1}=v_{k}-\left[F\left(2 v_{k}-x_{k} ; x_{k}\right)\right]^{-1} F\left(v_{k}\right), \\
v_{k}=x_{k}-\left[F\left(2 v_{k-1}-x_{k-1} ; x_{k-1}\right)\right]^{-1} F\left(x_{k}\right) .
\end{gathered}
$$

Этот метод удобен в реализации, поскольку требует вычисления на каждом шаге итерации (за исключением первого) лишь двух значений оператора и одной первой разделенной разности.

3 а меч ан ие. Отметим, что соответствующий основной метод при $\varrho \rightarrow 0$ можно получить из метода Ньютона

$$
x_{k+1}=x_{k}-\Gamma_{k} F\left(x_{k}\right),
$$

если его преобразовать к виду

$$
x_{k+1}=x_{k}+\left(\Gamma_{k} F^{\prime}\left(x_{k}\right)-2 I\right) \Gamma_{k} F\left(x_{k}\right)
$$

и заменить в нем $x_{k}$ на $v_{k}=x_{k}-\Gamma_{k} F\left(x_{k}\right)$ всюду, кроме $\Gamma_{k}$. Известно $\left[{ }^{8}\right]$, что при подстановке $v_{k}$ прямо в метод Ньютона получаемая комбинация дает порядок сходимости, равный лишь трем.

2. Если принять $\varrho=-1$ и $A_{k}=\mathscr{A}_{k}=\Gamma_{k}$, то из (2) получим итерационный метод четвертого порядка сходимости: 


$$
x_{k+1}=v_{k}-\Gamma_{k} F\left(v_{k}\right)-\Gamma_{k} F\left(v_{k}-\Gamma_{k} F\left(v_{k}\right)\right) .
$$

Положим $Q_{1}(x)=x$. Тогда

$$
Q_{2}(x)=Q_{1}(x)-\Gamma(x) F\left[Q_{1}(x)\right]=x-\Gamma(x) F(x)
$$

порождает метод второго порядка сходимости - метод Ньютона, и в общем случае $(p \geqslant 2)$

$$
Q_{p+1}(x)=Q_{p}(x)-\Gamma(x) F\left[Q_{p}(x)\right]
$$

порождает метод $(p+1)$-порядка сходимости $\left[{ }^{8}\right]$ :

$$
x_{k+1}^{(p+1)}=Q_{p+1}\left(x_{k}\right)=Q_{p}\left(x_{k}\right)-\Gamma\left(x_{k}\right) F\left[Q_{p}\left(x_{k}\right)\right] .
$$

При $p=3$ получим метод (6).

Рассмотрим теперь беспроизводный аналог метода (7), т. е. вместо $\Gamma_{k}$ будем использовать некоторую его аппроксимацию $A_{k}$. Оценка для этого варианта аналогична оценке (3):

$$
\begin{gathered}
\| F\left(\underset{k+1}{\left.x_{k+1)}^{(p+1)}\right) \| \leqslant} \gamma_{k}^{p}\left\|F\left(x_{k}\right)\right\|+K_{p-1}^{(p+1)} \gamma_{k}^{p-1}\left\|F\left(x_{k}\right)\right\|^{2}+\ldots\right. \\
+K_{0}^{(p+1)}\left\|F\left(x_{k}\right)\right\|^{p+1}+R^{(p+1)}
\end{gathered}
$$

где $R^{(p+1)}$ содержит члены более высокого порядка, т. е. члены вида $O\left(\gamma_{k}^{s}\left\|F\left(x_{k}\right)\right\|^{t}\right), s+t \geqslant p+2, K_{s}^{(p+1)} \quad(s=0,1, \ldots, p-1)$ - постоянные величины. В самом деле, если вторая производная $F(x)$ ограничена, то при $p=1$ имеем

$$
\begin{gathered}
\left\|F\left(x_{k+1}^{(1)}\right)\right\| \leqslant \|[ \\
\times\left[F_{k} F\left(x_{k}\right)\right]^{2}\left\|\leqslant \gamma_{k}\right\| F\left(x_{k}\right)\left\|+K_{0}^{(1)}\right\| F\left(x_{k}\right) \|^{2} .
\end{gathered}
$$

Предположим теперь, что

$$
\left\|F\left(x_{k+1}^{(p)}\right)\right\| \leqslant \gamma_{k}^{p-1}\left\|F\left(x_{k}\right)\right\|+K_{p-2}^{(p)} \gamma_{k}^{p-2}\left\|F\left(x_{k}\right)\right\|^{2}+\ldots+K_{0}^{(p)}\left\|F\left(x_{k}\right)\right\|^{p}+R^{(p)} .
$$

Тогда

$$
\begin{aligned}
\left\|F\left(x_{k+1}^{(p+1)}\right)\right\| & =\| F\left(x_{k+1}^{(p)}\right)-F^{\prime}\left(x_{k+1}^{(p)}\right) A_{k} F\left(x_{k+1}^{(p)}\right)+ \\
& +\int_{0}^{1} F^{\prime \prime}\left(x_{k+1}^{(p)}-\tau A_{k} F\left(x_{k+1}^{(p)}\right)\right)(1-\tau) d \tau\left(A_{k} F\left(x_{k+1}^{(p)}\right)\right)^{2} \| \leqslant \\
& \leqslant\left\|\left[I-F^{\prime}\left(x_{k+1}^{(p)}\right) A_{k}\right]\right\|\left\|F\left(x_{k+1}^{(p)}\right)\right\|+G\left\|F\left(x_{k+1}^{(p)}\right)\right\|^{2} .
\end{aligned}
$$

Поскольку

$$
F^{\prime}\left(x_{k+1}^{(p)}\right)=F^{\prime}\left(x_{k}\right)+\int_{0}^{1} F^{\prime \prime}\left(x_{k}+\tau\left(x_{k+1}^{(p)}-x_{k}\right)\right) d \tau\left(x_{k+1}^{(p)}-x_{k}\right)
$$

и

$$
\left\|x_{k+1}^{(p)}-x_{k}\right\| \leqslant E\left\|F\left(x_{k}\right)\right\|+R_{1},
$$

где $E-$ постоянная, $R_{1}$ содержит произведения величин $\gamma_{k}$ и $\left\|F\left(x_{k}\right)\right\|$ и их степеней, то нетрудно получить из равенства (9) оценку (8).

Если для построения $A_{k}$ воспользоваться итерационной формулой

$$
A_{k}=A_{k-1}\left(I+E_{k-1}+E_{k-1}^{2}+\ldots+E_{k-1}^{q-1}\right) \quad(k=0,1, \ldots),
$$


где $E_{k-1}=I-F^{\prime}\left(x_{k}\right) A_{k-1}$, то с учетом (8) можно доказать, что при $q \geqslant p+1$ метод (7) сохраняет свой порядок сходимости $p+1$, что согласуется также с результатами работы : $\left[{ }^{9}\right]$.

3. Изложенное позволяет сделать вывод, что сохранение четвертого порядка сходимости для класса методов (2) обеспечивается, если соответствующие им линейные уравнения (4) решать с точностью $O\left(\left\|F\left(x_{k}\right)\right\|\right)=O\left(\left\|F\left(x_{0}\right)\right\|^{4^{k}}\right)$, а для метода (7) - с точностью $O\left(\left\|F\left(x_{0}\right)\right\|(p+1)^{k}\right)$, т. е. требования к точности решения линейных проблем увеличиваются с ростом порядка сходимости.

Таким образом, при оценке эффективности по критерию, использующему так или иначе порядок сходимости и объем вычислений, более реальную картину мы получим, если в качестве порядка $p$ будем принимать не теоретический порядок рассматриваемого метода, а порядок, который мы сможем ему обеспечить путем выявления погрешностей, возможных при его практической реализации. Отметим, что к ошибкам вычислений наиболее чувствительны методы высокого порядка сходимости.

\section{Л И Т Е Р А Т У Р А}

1. Островский А. M., Решение уравнений и систем уравнений, M., 1963.

2. Tra ub, J. F., Iterative Methods for the Solution of Equations, New York, 1964.

3. Б а р тіш М. Я., Докл. АН УРСР, Сер. физ.-техн. и матем. наук, 5, 387 (1968).

4. S c h m i d t, J. W., Le de r, D., Computing, 5, 71 (1970).

5. В а а р м анн О., Полль В., № 140-76 Деп., Таллин, 1975.

6. С ер ге в А. С., Сиб. матем. ж., 2, № 2, 282 (1961).

7. S ch m idt, J. W., Z. angew. Math. und Mech., 43, Nr. 3, 97 (1963).

8. Щ е р би а Ю. Н., Автореф. канд. дисс., Львов, 1975.

9. Гор фунжель И. А., Вычисл. и прикл. матем., Межвед. научн. сб., вып. 26. 135 (1975).

Ннститут кибернетики

Академии наук Эстонской ССР
Поступила в редакцию 30/VI 1976

O. VAARMANN, V. POLL

\section{MITTELINEAARSETE VŌRRANDITE LAHENDAMISEST KÖRGET JÄRKU KOONDUVUSKIIRUSEGA ITERATSIOONIMEETODITEGA JA NENDE STABIILSUSEST}

Analüüsitakse arvutusvigade mõju koonduvuskiiruste hinnangutele ühe neljandat järku koonduvuskiirusega iteratsioonimeetodite klassi näite varal. On vaadeldud juhtu, kus pöördoperaatori asemel kasutatakse mingit tema aproksimatsiooni. Viimane asjaolu on ekvivalentne sellega, et vastav lineaarne operaatorvõrrand lahendatakse teatava veaga. Näidatakse, et mida kõrgemat järku on meetod, seda täpsemalt tuleb järgu sáilivuse garanteerimiseks lahendada lineaarseid vőrrandeid. Erijuhul on saadud vaadeldavasse klassi kuuluvate iteratsioonimeetodite tuletisvabad analoogid ja hinnatud nende koonduvuskiirusi.

\section{O. VAARMANN, V. POLL}

\section{ON SOLVING NONLINEAR EQUATIONS BY ITERATIVE METHODS OF HIGH-ORDER CONVERGENCE AND THEIR STABILITY}

In this paper an error analysis of iterative methods of high-order convergence is outlined. To discuss the numerical problems associated with solving linear equation approximately at every iteration, the case is considered where instead of the inverse operator an approximation is used. It is shown that the higher order of convergence, the more accurately, in general, the related linear equations are to be solved to preserve the order of convergence. In particular, the derivative-free analogues of these methods are obtained, and their rates of convergence estimated. 